This item was submitted to Loughborough's Research Repository by the author.

Items in Figshare are protected by copyright, with all rights reserved, unless otherwise indicated.

\title{
Mechanical characterization of hydrogels and its implications for cellular activities
}

PLEASE CITE THE PUBLISHED VERSION

http://dx.doi.org/10.1039/9781782622055-00171

\section{PUBLISHER}

(C) the authors. Published by the Royal Society of Chemistry (RSC)

\section{VERSION}

AM (Accepted Manuscript)

\section{PUBLISHER STATEMENT}

This work is made available according to the conditions of the Creative Commons Attribution-NonCommercialNoDerivatives 4.0 International (CC BY-NC-ND 4.0) licence. Full details of this licence are available at: https://creativecommons.org/licenses/by-nc-nd/4.0/

\section{LICENCE}

CC BY-NC-ND 4.0

\section{REPOSITORY RECORD}

Wilson, Samantha L., Mark Ahearne, Alicia J. El Haj, and Ying Yang. 2019. "Mechanical Characterization of Hydrogels and Its Implications for Cellular Activities”. figshare. https://hdl.handle.net/2134/34699. 


\section{Mechanical Characterization of Hydrogels and its Implications for Cellular Activities}

2

3 Samantha L Wilson ${ }^{\mathrm{a}, \mathrm{b}}$, Mark Ahearne ${ }^{\mathrm{c}}$, Alicia J El Haj ${ }^{\mathrm{a}}$ and Ying Yang*a

4

5

6 a Institute of Science and Technology in Medicine, School of Medicine,

$7 \quad$ Keele University, Stoke-on-Trent, ST4 7QB, UK

8 bophthalmology, Division of Clinical Neurosciences, University of Nottingham, Queen’s

9 Medical Centre Campus, NG7 2UH, UK

10 'Trinity Centre for Bioengineering, Trinity Biomedical Sciences Institute, Trinity College Dublin,

11 Ireland

12

13 *Corresponding author: Ying Yang, E-mail: y.yang@keele.ac.uk 


\section{Introduction}

17 One of the challenges faced by scientists and clinicians is to fabricate physiologically

18 relevant three-dimensional (3D) culture models with controllable biochemical and biophysical properties that can provide an in vitro platform to develop and test new clinical therapies.(1) The use of hydrogels is among some of the more promising approaches for the

21 development of culture models for use in tissue engineering and regenerative medicine.

22 These biomaterials consist of a water-swollen network of crosslinked hydrophilic polymer 23 chains. The limited availability of native tissues for transplantation and in vitro testing has propelled the need to develop new hydrogels that replicate native tissue extracellular matrix (ECM). Hydrogel materials can be fabricated from natural protein polymers such as collagen, fibrin, agarose, gelatine or alginate, or from synthetic polymers such as poly(ethylene glycol)

27 (PEG), poly(vinyl acetate) (PVA) or poly(acrylic acid) (PAA). The choice of polymer is vital when determining the suitability of a particular hydrogel material for a given application.(2) Natural protein hydrogels are advantageous in that they provide native biochemical cues and are able to simulate many aspects of the natural ECM. Synthetic hydrogels are valuable in that more well-defined, easily tuneable structures and mechanical properties can be achieved in comparison to protein-based hydrogels. Frequently a combination of natural and synthetic hydrogels is utilized in order to more closely mimic the dynamic native culture environments that change in response to cellular behaviour. Hydrogels provide a popular method of culturing cells in a 3D environment as they provide a structure in which a tissue can develop. Hydrogels act as a temporary matrix that allows cells to grow, move and communicate. Their viscoelastic characteristics, biocompatibility, availability and their ability to be remodelled by cells make them a suitable material for tissue regeneration. Cell-seeded hydrogel constructs can also replicate the close contact/adhesion that occurs between cells and ECM. Hence the mechanical properties of the resulting hydrogel construct become a unique property that 
41 mutually affects the constructed hydrogel and the cells. Characterization of the mechanical properties of hydrogel constructs may ultimately have implications on cellular actives.

43 In vivo and in vitro the extracellular environment is vital in controlling cell heath and 44 provides both chemical and mechanical stimuli that influence cellular behaviour.(1,3) In vivo cells are organized into tissues and organs with complex mechanical and structural architectures.(3) Both endogenous and exogenous forces act upon the cells and their

47 surrounding environment. Endogenous factors include cell-integrin binding to the ECM and 48 cellular responses to soluble factors such as growth factors and cytokines. Exogenous forces include gravity, substrate stiffness, polarity and surface to volume ratios (1) and tissuespecific interactions including traction forces generated by cells.(4)

51 Many cell types can be described as 'anchorage dependent' in that, to remain viable, they require a substrate to attach to.(5) Most soft tissues including vascular, cardiac, dermal,

53 muscle, brain, tendon and cornea consist of ECM combined with adherent cells that possess

54 elastic or visco- elastic characteristics(5) in vivo. Tissue culture plastic and glass coverslips 55 provide a relatively rigid microenvironment (lacking many mechanical and biophysical cues) 56 for cells to be cultured in vitro when compared to cells in native tissues. In vivo it is the 57 combination of the cellular microenvironment and chemical and physical cues that mediate 58 cellular behaviour. These niche environments are often very difficult to replicate in vitro.

59 Cellular behaviour can vary markedly based upon the mechanical properties of the culture 60 substrate.(6) Cellular migration, adhesion, proliferation, migration and differentiation can all 61 affect and be affected by the mechanical properties of a tissue.(3) 


\section{Hydrogel Characterization Techniques}

64 The mechanical and viscoelastic properties of hydrogel materials are important parameters

65 when considering their suitability for use with cells and tissues. These same properties are highly reliant on environmental factors such as temperature and $\mathrm{pH}$, in addition to the cell activity. Thus, it is imperative to be able to determine the material properties of hydrogel constructs under conditions similar to the in-situ environment in which they will be utilized.(2) Many techniques exist to measure the mechanical properties of hydrogels and are centred on theories of rubber elasticity and viscoelasticity.(2) In general, hydrogels can be considered to behave in a viscoelastic manner, meaning they exhibit both viscous and elastic characteristics. The relationship between stress and strain in viscoelastic materials is dependent on time. Frequently used methods for measuring the mechanical properties of

74 hydrogels include tensile, compression or indentation techniques.

75 Tensile testing or strip extensiometry testing are the most frequently used methods for the mechanical characterization of hydrogels.(7) This test involves clamping the hydrogel

77 between two grips and then stretching it (Figure 1A). The amount of force required to stretch

78 the hydrogel is measured and plotted against the distance the hydrogel has been stretched.

79 The force and distance can be used to determine the stress and strain applied to the hydrogels and from this the Young's modulus can be calculated. Other parameters such as the ultimate tensile strength and yield strength of the hydrogels may also be determined using this test

82 although these would require testing the sample to failure. This test can also be used to 83 determine the viscoelastic characteristics of the hydrogel by stretching the hydrogel a predetermined distance and measuring the change in force required to maintain that elongation over time. If the hydrogel is viscoelastic, it should undergo stress relaxation resulting in a reduction in force over time until reaching equilibrium. The dynamic modulus

87 of the hydrogels may also be determined by repetitively loading and unloading of the 
hydrogel. A variation on the standard tensile test involves using a hydrogel ring that is stretched between two posts. The advantage of this approach is that no grips are required. For both approaches hydrogels may be immersed in solution to ensure that they are maintained in a swollen state. The principle advantage of using these tests are their relative simplicity compared to other techniques. One limitation is that in general only uniform strips or rings can be tested; more complicated geometries would require more complex mechanical models to calculate values. In addition, the fragility of hydrogels can make them difficult to handle and grip in this system.

Compression testing has also been used to examine the mechanical properties of hydrogels.

For this technique the hydrogel is placed under a uniform load that results in the hydrogel being compressed (Figure 1B). Depending on how the system is set up, either the load or the distance can be controlled while the other is measured. The resulting stress-strain relationship can be used to calculate the compressive modulus of the hydrogel. Due to the viscoelastic nature of hydrogels, typically dynamic moduli at specified frequencies or equilibrium modulus are determined. The equilibrium modulus is calculated from the stressstrain data after the hydrogel has undergone stress relaxation. This technique has previously been used to measure the mechanical properties of several types of cell-seeded hydrogels including fibrin, agarose and gellan gum hydrogels.(8) The samples can be fully submerged in solution during testing to prevent dehydration. Unlike extensometry, the geometry of the hydrogel is not limited to strips or rings, although a flat surface is required. Usually cylindrical hydrogel constructs are used. Limitations include bulging of the material and difficulties in applying even pressure to the sample.

A bulge or inflation test is a more novel technique where a hydrogel can be characterized by inflating it. The hydrogel is held in a sample holder and fluid is pumped underneath it causing it to bulge (Figure 1C). The bulge displacement as a function of the applied fluid pressure is 
113 measured using a charge-coupled device (CCD) camera or a laser.(7) The relationship

114 between the applied pressure and the resultant strain on the hydrogel can be incorporated into

115 a mathematical model to calculate the elastic or viscoelastic properties of the hydrogel.

116 Leakage, difficulties controlling and measuring the applied pressure and dissolved air

117 becoming trapped in the solution are all problems associated with bulge and indentation

118 testing. The test is also only suitable for flat uniform hydrogels.

119 Indentation techniques have been widely used to characterize soft biomaterials including

120 hydrogels. Hayes et al. (9) were one of the first groups to use indentation to examine the

121 mechanical properties of a tissue. They used indentation to examine the mechanical

122 properties of human cartilage. Indentation has also been used to examine the adhesive

123 characteristics of tissues (10) by measuring the adhesion force between the indenter and the

124 tissue. There are several variations of the indentation techniques used to characterize

125 hydrogels including spherical indentation, micro-indentation and nano-indentation.

126 Spherical indentation involves suspending a thin circular hydrogel around its outer

127 circumferences in a specifically designed sample holder and placing a ball of known weight

128 and size onto the hydrogel (Figure 1D). The weight of the ball causes the hydrogel to

129 deform. The deformation is recorded via a CCD camera and the depth of indentation is used

130 to calculate the elastic modulus of the hydrogel.(11) The viscoelastic properties of the

131 hydrogel can also be monitored by measuring the change in central deformation over

132 time.(12) This approach is particularly suitable for cell-seeded hydrogels as the whole

133 assembly can be fully submerged in solution and be kept in an incubator at $37^{\circ} \mathrm{C}$ while

134 testing. This technique has been used to examine the effect of fibroblasts on the mechanical

135 properties of collagen hydrogels (13) and the influence of nano fibres on hydrogel properties,

136 (14) and for optimizing crosslinking conditions for hydrogels.(15) This technique allows for 
137 online, real-time and non-destructive measurements to be taken over prolonged culture 138 periods.

139 A variation of the spherical indentation technique involves placing a hydrogel onto a flat 140 substrate rather than suspending it (Figure 1E). This approach is more suitable to thicker 141 hydrogels while the suspension approach is more suited to thinner hydrogels. The weight of

142 the ball causes the hydrogel to deform and the deformation depth and weight of the ball can

143 be applied to mathematical model called the Hertz model, to calculate the elastic modulus of

144 the hydrogel. The main difficulty with this technique is accurately measuring the depth of

145 indentation. One method around this problem is to use optical coherence tomography (OCT).

146 The combination of spherical indentation and OCT has previously been used to measure the

147 mechanical properties of agarose hydrogels.(16)

148 Micro-indentation involves deforming a hydrogel using a rigid indenter connected to a force 149 transducer. The indenter is lowered onto the hydrogel and deforms it to a particular depth.

150 The depth of indentation and the amount of force applied are both applied to theoretical

151 model to calculate the mechanical properties of the hydrogel. The hydrogel may be suspend 152 around it outer edge or placed flat on a substrate (Figure 1F and G). For suspended 153 hydrogels a number of different theoretical models can be used to calculate the mechanical 154 properties of the hydrogel.(17) For hydrogels on a flat substrate, the Hertz model is used to 155 calculate the modulus of the hydrogel.(18) Micro-indentation can be used to examine 156 regional variation across different areas of a hydrogel.

157 Nano-indentation works on the same principle as micro-indentation, but the tip size and 158 indentation depth are on a nanometric scale. This apparatus consists of a sharp-tipped 159 indenter attached to a cantilever beam. Mechanical characterization at this scale is limited to 160 producing data on the surface properties of the hydrogel. The difficulties associated with 
161 using nano-indentation with hydrogels include accurate calibration of the instrument, applying a suitable mechanical model and the elimination of other sources of error.(19)

163 Eastwood et al.(20) developed a tensioning-culture force monitor system which can apply a 164 predetermined loading to the hydrogel, in particular collagen hydrogel, and monitor the contraction strain generated by resident fibroblasts. The beauty of this system is that it can monitor the strain development for days continuously and visualize the associated global 167 morphology change.

168 Ultrasound elastography is a technique that works by transmitting ultra- sonic waves through 169 the hydrogel and then reading backscattered waves, which can be used to form 2D images.

170 When a force is applied to the hydrogel, the resulting displacement can be detected

171 throughout the hydrogel. This information can then be processed to characterize the 172 mechanical properties of the hydrogel. Fromageau et al.(21) used several variations of this 173 technique to measure the Young's modulus of PVA hydrogels. They found that elastography 174 produced similar mechanical values to standard mechanical testing techniques. The main

175 limitation with this technique is the costs involved in the purchasing and running of the 176 ultrasound equipment.

177 Recently, Li et al.(22) explored a novel approach that utilizes a low-coherence interferometer 178 to detect the laser-induced surface acoustic waves (SAW) from agar hydrogels to mimic soft 179 tissues. This technique allows for rapid characterization of the elastic properties of soft 180 biological tissues and has the advantage of being a non-destructive technique.

181 There is a widespread demand for the development of non-destructive techniques that permit 182 the continuous measurement of hydrogel constructs for prolonged culture periods. The use of 183 non-destructive mechanical characterization techniques is extremely valuable in that they 184 allow for changes in mechanical properties over time to be characterized. Such changes can 
185 then be more accurately linked to cell activity and remodelling of the hydrogel matrix.

186 Among other techniques, micro-indentation, ultrasound elastography and the combination of

187 OCT and surface acoustic wave or with indentation techniques are extremely powerful tools

188 for the characterization of the mechanical properties of hydrogels or soft tissues.

\section{Effect of Hydrogel Mechanical Properties on Cellular Activities}

191 In most native tissues, anchoring cells attach to the surrounding ECM. This ECM provides an inner physical support and its composition, topography and stiffness provides biochemical and biophysical cues that are necessary to the development and maintenance of these tissues.

194 Until recently, chemical regulators within the extracellular environment have primarily been investigated, with little emphasis regarding the influence of mechanical regulation.(3) Similar to surface chemistry, the mechanical properties affect the local behaviours of tissues and cells. Normally cells embedded in tissues are able to 'probe', 'feel' or 'sense' the elasticity or stiffness of their surrounding matrix $(5,6)$ or substrate as they anchor and pull themselves along during cell migration. 'Stiffness' refers to the measure of a material's ability to resist deformation and this can change during physiological processes including embryonic development, wound healing and pathological conditions.(4) In the body, the magnitude of stiffness is vast, ranging from a few $\mathrm{kPa}$ in adipose tissue23 to GPa in bone.(4,24) A wide variation in matrix stiffness along with biochemical signals influence focal-adhesion

204 structures and the cytoskeleton.(5) Previous studies using cells committed to a particular 205 lineage, especially fibroblasts, on coating collagen gels and wrinkling-silicone sheets also suggest some responsiveness to the physical state of the matrix.(25)

207 In addition to applying force to its surroundings, the cells themselves respond to the 
be altered during pathological conditions, this can affect cellular behaviour and

211 morphology in response to changes in substrate resistance or stiffness.(5) For example, cells

212 attached to stiff, rigid constructs will form stable focal adhesions, whereas cells attached to

213 less stiff materials will have diffuse and dynamic adhesion complexes.(5,26 )This can have a

214 direct impact on cellular migration and proliferation, such as increased proliferation in cells

215 seeded onto stiffer substrates.(6) This can be linked to cellular wound healing responses as,

216 often, the granulation and change in mechanical properties of scar tissue (6) is related to

217 cellular infiltration and remodelling. In general, cells appear to adhere, spread and survive

218 better on stiffer materials, although there are exceptions to this including neutrophils, which

219 do not appear to be affected by substrate stiffness,(27) and neurons, which actually show

220 improved survival on stiffer materials.(28) Studies on fibroblasts cultured on hydrogels have

221 demonstrated that substrate stiffness significantly alters ECM assembly, cell spreading and

222 motility.(6,29)

223 The mechanical properties of hydrogels can have a profound influence on regulating the

224 phenotypic behaviour of cells. This is most noticeable with stem cells, where variations in

225 stiffness can promote differentiation towards different lineages. Engler et al.(30) showed that

226 the ability of stem cells to differentiate towards specific lineages was dependent on the

227 substrate stiff- ness of the materials on which the cells were cultured. They noted that

228 neurogenic differentiation was optimal at a stiffness of $0.1-1 \mathrm{kPa}$, myogenic differentiation at

229 8-17 kPa and osteogenic differentiation at 25-40 kPa. In hydrogels the effect of the

230 concentration, which is directly linked to mechanical properties, on the differentiation of

231 neuronal stem has been investigated.(31) Phenotypic neuronal markers were up regulated

232 when the hydrogel stiffness matched that of brain tissue. Bian et al.(32) found that the

233 chondrogenic capacity of mesenchymal stem cells could be optimized by varying the stiffness 
234 of hyaluronic acid hydrogels. Likewise, Steward et al.(33)found that differentiation of

235 mesenchymal stem cells could be partially regulated by hydrogel stiffness towards osteogenic

236 or chondrogenic lineages.

237 Cell adhesion can also play a significant role on the mechanical properties of hydrogels and is

238 vital for many other processes such as matrix contraction and cell migration. Hydrogel

239 stiffness is dependent on the polymer concentration and the crosslinking density. A

240 consequence of increasing the polymer concentration often results in a subsequent increase in

241 adhesion sites. Cell adhesion can have a profound effect on cell behaviour and cell

242 phenotype. Trappmann et al.(34) showed that differing protein anchorage densities can be

243 used to regulate stem cell fate. This finding is important as it demonstrates the interdependent

244 relationship between matrix stiffness, binding site availability and cell phenotype. Of course,

245 not all hydrogels facilitate binding by cells. Steward et al.(35) compared the phenotypic and

246 cytoskeletal behaviour of cells in a hydrogel that facilitates binding, i.e. fibrin, and cells in a

247 hydrogel that lack binding sites, i.e. agarose. Cells in hydrogels that allowed binding had a

248 spread morphology while those in hydrogels that lacked binding sites maintained a spherical

249 morphology.

\section{4. Effect of Cellular Activity on Hydrogel Properties}

251 In addition to hydrogels having an effect on the behaviour of cells, reciprocally the cell

252 activity within the hydrogels can affect their bulk mechanical properties. Similar phenomena

253 are found in diseases such as those that affect connective tissues and alter the mechanical

254 properties of the tissue. The result is often the formation of hard tumours or the generation of

255 ulcers. In these cases, it is the cellular activities that regulate the ECM. Hence, cells seeded in

256 hydrogels can affect their mechanical properties through several metabolic activities 
including digestion via the production of enzymes; proliferation; matrix synthesis; contraction; ECM deposition and crosslinking.

259 Enzymes are produced by cells to initiate matrix remodelling and cell migration. Among the most prominent enzymes produced by cells are a family of enzymes called matrix metalloproteinases (MMPs). Mauch et al.(36) showed that fibroblasts seeded in collagen hydrogels release MMPs, resulting in the degradation of the hydrogel matrix. The enzymatic degradation of hydrogels by cells can be easily controlled by the addition of MMP inhibitors and other reagents that can influence the cell-matrix mechanical relation- ship. In addition to preventing matrix degradation, the inhibition of MMPs may also prevent the contraction and remodelling of hydrogels. Ahearne et al.(13) found that exogenous addition e.g. cytochalasin, to fibroblast-seeded collagen hydrogels reduced the fibroblasts' contractibility and capacity of adhesion to hydrogel, which significantly decreased the elastic modulus of 269 the hydrogel construct (Figure 2).

270 One of the most prominent mechanisms by which cells affect the mechanical properties of 271 hydrogels is through the production of ECM proteins. For example, hydrogels seeded with 272 chondrocytes, mesenchymal stem cells or infrapatellar fat-pad derived progenitor cells have 273 been shown to increase the stiffness of these hydrogels when they were cultured in a 274 chemically defined prochondrogenic medium.(8,37-39) This increase in stiffness has been 275 attributed to the release of collagen and sulfated glycosaminoglycans (sGAG) by cells that 276 accumulate in the hydrogel and can be determined using biochemical analysis and

277 histological staining, as shown in Figure 3. Hydrogels may also undergo calcification when 278 cells are cultured in a pro-osteogenic medium. The increase in calcium deposition can 279 increase the bulk stiffness of the hydrogel. Calcium phosphate may also be incorporated into 280 the hydrogel prior to fabrication to increase the hydrogel stiffness or induce it to be formed. 281 Douglas et al.(40) incorporated alkaline phosphatase into collagen and PEG-based hydrogels 
to induce their mineralization into calcium phosphate. They found that calcium phosphate was formed, and mineralization increased the Young's modulus of the hydrogel. One factor that needs to be considered when relating hydrogel stiffness to ECM production is the ability of the hydrogel to retain the newly formed matrix components. Hydrogels with a high porosity or high-water content may not retain matrix proteins as easily as other hydrogels. The loss of matrix proteins in this manner would affect the change in hydrogel stiffness. Many hydrogels undergo contraction when seeded with cells (Figure 4). Hydrogels such as collagen and fibrin contain ligands that enable cell adhesion. Due to their limited mechanical strength, the cells are able to contract these hydrogels. Contraction can be a problem when attempting to design hydrogels that incorporate specific geometries that replicate the native tissues. These geometries can be destroyed by cells contracting their surrounding matrix.(41) Bell et al.(42) have suggested that contraction can be advantageous and may be used to enable the cells to engineer hydrogels into tissue-like structures. It has previously been shown that increasing the stiffness of these hydrogels reduces the rate of contraction. Ahearne et al.(43) showed that following 25 days in culture, collagen hydrogels seeded with fibroblasts at a concentration of $2.5 \mathrm{mg} \mathrm{mL}^{-1}$ reduced in thickness by $85 \%$ as a result of contraction, while stiffer hydrogels fabricated at a concentration of $4.5 \mathrm{mg} \mathrm{mL}^{-1}$ showed a reduced in thickness by approximately $60 \%$. their surrounding matrix.(44) The application of force onto cells can initiate several cellular processes including ion-channel activation, phosphorylation and cytoskeletal changes.

303 Another factor that plays an important role in dictating the cell-matrix mechanical relationships is the initial conditions used to manufacture the hydrogels. Cell seeding density can also influence the mechanical properties of hydrogels. Increasing the cell number often leads to an increase in the rate of hydrogel remodelling and changes in mechanical properties. 
307 For fibroblast-seeded collagen hydrogels, the initial cell and collagen concentration used was 308 found to affect the ability of cells to change the mechanical properties of the hydrogels.(43)

309 Varying the initial cell density in turn varies the amount of force that can be generated to

310 remodel the hydrogels. This phenomenon demonstrates a clear mechano feedback response

311 between firoblasts and their surrounding hydrogel matrix.

\section{5. Mechanical Properties as a Marker of Cellular Activities}

313 The mechanical properties of soft tissues are often closely related to their physiological

314 function. For example, in vivo tissue contraction, remodelling and fibrosis (or scarring)

315 following injury or disease often results in an alteration to the mechanical properties of the

316 affected tissue (45) due to an 'activation' of the native cell phenotypes into their injury

317 subtypes.(46) The dynamic reciprocity between hydrogel mechanical properties and cell

318 activity has driven researchers to investigate how the mechanical properties of hydrogel

319 constructs affects cell behaviour and whether these properties can act as a marker to predict

320 cellular activity. This relationship has to be considered when designing hydrogels that need to

321 be suitable for implantation. Here we present several examples to demonstrate how the

322 mechanical properties of hydrogel constructs can reflect cellular activities.

323

324 5.1. Indicator of Differentiation Status

325 A recent study by Wilson et al.(14) demonstrated how the assessment of mechanical

326 properties in terms of elastic modulus measurement could be used to determine the effect of

327 biochemical ingredients and topographic features on corneal stromal cell differentiation in

328 collagen hydrogels. The aim was to determine the most suitable culture condition whereby

329 cultured corneal stromal cells that were initially 'activated or fibroblastic in phenotype could 
330 be restored to the native 'inactivated' keratocyte phenotype in vitro. The basis of using matrix contraction capacity as an additional marker of corneal stromal cell phenotype differentiation in response to different culture conditions is that corneal keratocytes are quiescent and

333 noncontractile and corneal fibroblasts are contractile and motile.(47) Thus, the construct

334 contraction and elastic modulus measurements combined provided a descriptive insight into 335 what was happening at a cellular level within the constructs when culture conditions change.

336 This was used to indicate that the synergistic effect of nanofibre incorporation, serum

337 removal, plus insulin medium supplementation provided the most suitable environment for

338 the restoration of the native corneal keratocyte cell phenotype. These results were then

339 corroborated with microscopic and genotypic characterization data to further validate that

340 mechanical characterization can act as a sensitive marker of cellular activities such as cell

341 phenotype and differentiation (Figure 5).

\subsection{Indicator of Cell Viability and Contractility}

344 Ahearne et al.(13) used a spherical indentation technique to investigate the relationship

345 between cell viability, hydrogel contraction and hydrogel elastic modulus in response to long-

346 term culture (Figure 6). It was found that an initial increase in elastic modulus coincided with

347 contraction of the hydrogel while a reduction in cell viability over several weeks in culture

348 resulted in a reduced modulus. Inhibition of contraction using an MMP inhibitor found that

349 when contraction was prevented, there was no subsequent increase in modulus. It was also

350 found that the inhibition of actin stress fibres resulted in a reduction in elastic modulus,

351 suggesting that the intrinsic strain applied by these cells was instrumental in controlling the

352 bulk mechanical properties of the hydrogel. The actin staining images at corresponding time

353 points exhibited clear morphology difference in responding to the associated modulus the 
specimens exhibited higher modulus expressed highly stretched actin filaments (Figure 6B and $\mathbf{C}$ ), while the destroyed actin morphology (Figure 6D) appeared at the specimens which had low modulus with long culture duration, implying the low cell viability

\subsection{Indicator of Network Structure in the Hydrogel}

359 There have been a large number of reports dedicated the effect of ageing on protein structures; in particular collagen type I, as it is a key lifelong structural protein in the body. A prevalent ageing mechanism, concerned with the non-enzymatic glycation of collagen, is the formation and accumulation of advanced glycation end-products (AGEs).48 Accumulation of

363 AGEs in relation to increasing chronological age has been linked to permanent alterations to the intra- and intermolecular structure of collagen, which often manifests as compromised mechanical properties to the tissue or construct being investigated. In recent work by Wilson et al.,(49) type I collagen was extracted from the tendons of different aged rats, varying from 2-3 days (newborn) to 2 years (old adults). The mechanical properties of the resulting reconstituted hydrogel constructs were then measured using an indentation technique.(50) It was found that in acellular hydrogel scaffolds that there was a clear visible trend showing that increasing age resulted in a reduced in the elastic modulus (Figure 7). The preliminary examination of the elastic modulus of corneal stromal fibroblasts grown in these hydrogels

372 found that younger collagen induced higher contraction than older collagens manifesting as a

373 higher modulus. Hence, it has been postulated that at a given collagen concentration, the

374 younger collagen hydrogels (newborn and 2 months old) with a highly organized fibrous

375 structure, resulted in a higher construct modulus compared to the randomly and loosely

376 packaged older specimens (6 months and 2 years old). Thus, it is feasible to predict

377 microscopic differences in the collagen hydrogel through the measurement of mechanical 378 properties 
380 6. Strategies for Improving the Mechanical Properties of Hydrogels

381 When using hydrogels to study cell-ECM interactions, it becomes critical to tailor the

382 hydrogels' mechanical properties. Various strategies have therefore been proposed to

383 improve their mechanical characteristics. A fundamental limitation of hydrogels for tissue

384 engineering is their inferior mechanical strength and stiffness in comparison to the native

385 tissue that they are being used to replicate. These mechanical properties result from the high

386 water content and random fibre orientations found in hydrogels.(7) Once the mechanical

387 properties of a hydrogel material have been determined, it is often desirable to improve the

388 mechanical strength of the construct so that it is more suitable for a given application.(2) The

389 mechanical properties of hydrogels can be improved using numerous strategies including the

390 alteration of the co-monomer composition, increasing/decreasing the crosslinking density,

391 alterations to the conditions in which the polymer is formed,(2) the addition of cells onto or

392 into the matrix via matrix remodelling, ECM secretions and the application of intrinsic strain.

\subsection{Concentration}

395 One approach to improving the mechanical properties of hydrogels is to increase the polymer 396 concentration. Several studies have examined the relationship between mechanical properties 397 and polymer concentration in hydrogels. Ahearne et al.(50) found that there was an almost 398 linear increase in elastic modulus with hydrogel concentration when examining agarose and 399 alginate hydrogels. Buckley et al.(37) found a similar trend when measuring the equilibrium 400 and dynamic moduli of agarose hydrogels of increasing concentration. The elastic modulus of 
401 collagen hydrogels has also been shown to increase with concentration.(43) Interestingly, the

402 initial collagen concentration also affected the subsequent rate of hydrogel contraction and

403 matrix remodelling, with a lower initial collagen concentration having a faster rate of

404 contraction. This faster rate of contraction led to these hydrogels having a higher cell density

405 and a higher overall collagen density compared to the other hydrogels after 25 days in

406 culture.(43) Methods of increasing the concentration of hydrogels such as plastic

407 compression has also demonstrated the relationship between hydrogel stiffness and hydrogel

408 concentration.51,52 By pushing fluid out of the hydrogels, this led to an increase in

409 concentration thus an increase in stiffness. It has been reported that the polymer concentration

410 in these hydrogels can increase by a factor of over 100 .

411

$412 \quad$ 6.2. Crosslinking

413 Chemical and photochemical crosslinking of matrix components such as collagen can also be

414 used to ifluence the mechanical characteristics of hydrogels. Glutaraldehyde crosslinking of

415 hydrogels has been shown to enhance the mechanical strength of several types of

416 hydrogel.(53) The main problem with using glutaraldehyde is its toxicity. Alternative

417 crosslinking agents such as genipin have been suggested as these are less toxic than

418 glutaraldehyde.53 UVA-crosslinking in the presence of riboflavin has been shown to increase

419 the stiffness of collagen hydrogels without damage to the cells in those hydrogels.15 UV light

420 has also been used to develop hydrogels with a stiffness matrix gradient to allow for the study

421 of hydrogel stiffness and cell behaviour.(54)

422 
424 Altering the ratio of different monomers used to prepare a hydrogel is one of the simplest

425 methods to increase the mechanical properties of the construct.(2) Provided that the hydrogel

426 is not fabricated using identical monomer units, then by increasing the concentration of the

427 physically stronger component, this should give a favourable outcome. Alteration of the

428 polymerization conditions can dramatically alter the final formed product.(2) Time,

429 temperature and the amount and type of solvent used can all be altered accordingly. The

430 volume of solvent used is of particular importance since it can alter crosslinking density, the

431 type or nature of the solvent can alter the copolymer structure.(2) Post-polymerization

432 techniques can also alter the network structure of a hydrogel, causing alterations to

433 mechanical strength. In addition, thermal cycling of the polymer, which involves successive

434 freezing and thawing cycles can also increase the mechanical properties of hydrogels. $(2,55)$

435

436

\subsection{Orientation of Fibrous Components}

437 Often, the native tissue architecture is pivotal to the in vivo mechanical strength and function 438 of a tissue. Much research has focused upon the mimicking of native tissue architecture in 439 both 2D and 3D cultures. Contact guidance techniques have been extensively researched as 440 they affect several cell characteristics including orientation, morphology, differentiation and 441 secretion of ECM proteins. It is the material composition and more specically the 3D nano442 and microscale structure (the mesostructure) of bioartificial constructs that are pivotal to their 443 success.(51) Micro- and nanopatterned surfaces, magnetic alignment and electrospinning 444 techniques are among a variety of techniques utilized in order to achieve this. 
447 Micro- and nanopatterned surfaces are often manufactured by the use of templates with well-

448 defined groove widths and depths into which cells with and without matrix materials are

449 added.(56) The patterned surfaces effectively restrict random cell growth via the

450 incorporation of either physical or biochemical barriers. Orientated deposition of ECM

451 components is capable of reinforcing the substrate in a given direction, which enhances the

452 global mechanical properties of the original construct.(56)

453 6.6. Magnetically Aligned Collagen

454 Magnetic fields have been utilized in an attempt to create orientated collagen type I

455 fibrils.(57) The use of magnetic fields to induce collagen orientation is advantageous in that it 456 is non-destructive.(57) It has been reported that molecules of collagen can be assembled into 457 orientated fibrils via the application of a magnetic force.(57) In brief, this can be achieved by 458 loading an aliquot of collagen into a shallow sample holder and positioning it horizontally in 459 the central region of a split coil superconducting magnet and increasing the temperature from 46020 to $30{ }^{\circ} \mathrm{C}$ for approximately $30 \mathrm{~min}$. The collagen molecules assemble into orientated

461 fibrils perpendicular to the applied field and transform into a viscous gel that is stable and 462 orientated after the magnetic field is removed. A limitation of this technique is that fibril 463 diameter cannot be regulated using this technique. Furthermore, there is conflicting evidence 464 suggesting that the application of strong magnetic forces can in fact impair cell function and 465 viability.(58)

\subsection{Electrospinning of Nanofibres}

468 Electrospinning is a process that is able to produce continuous fibres from the submicron 469 down to the nanometre-diameter range.(59) These fibres can then be arranged to recreate the 
470 in vivo tissue microstructures and arrangements. Several studies have incorporated

471 electrospun aligned nanfibres into hydrogels to improve mechanical properties and regulate

472 cell behaviour. A schematic showing how aligned nanofibres meshes can be incorporated into

473 a collagen hydrogel is shown in Figure 8. Wilson et al.(60) found that there was an increase

474 in elastic modulus of collagen hydrogels seeded with corneal stromal cells after PLDLA

475 nanofibres were added. The nanofibers also influenced the cell phenotype and cell orientation

476 and reduced the rate of hydrogel contraction. Tonsomboon and Oyne (61) found a 10-fold

477 increase in modulus after incorporating crosslinked gelatine nanofibres into alginate

478 hydrogels. The combination of electrospun nanofibres and hydrogels represents an exciting

479 new approach to engineering tissues with improved mechanical properties.

480

\section{7. Conclusion}

482 It has been demonstrated that the mechanical properties of hydrogels play a key role in the 483 regulation of cellular activities and those cells are capable of remodelling the structural and 484 mechanical properties of their surrounding hydrogel matrix. Understanding this reciprocal 485 relationship is vital in the development of new tissue engineering and regenerative medicine 486 strategies. It is envisioned that, by tailoring the mechanical characteristics of hydrogels to 487 particular applications, more anatomically accurate tissues could be engineered. 
489 1. A. M. Kloxin, C. J. Kloxin, C. N. Bowman and K. S. Anseth, Adv. Mater.,2010, 22, 34844903494.

491 2. K. S. Anseth, C. N. Bowman and L. BrannonPeppas, Biomaterials, 1996, 17, 1647-1657.

492 3. B. Mason, J. Califano and C. Reinhart-King, in Engineering Biomaterials for Regenerative 493 Medicine, ed. S. K. Bhatia, Springer, New York, 2012, pp. 19-37.

494 4. J. D. Zieske, V. S. Mason, M. E. Wasson, S. F. Meunier, C. J. M. Nolte, N. Fukai, B. R. 495 Olsen and N. L. Parenteau, Exp. Cell Res., 1994, 214, 621-633.

496 5. D. E. Discher, P. Janmey and Y.-L. Wang, Science, 2005, 310, 1139-1143.

497 6. Y. Wang, G. Wang, X. Luo, J. Qiu and C. Tang, Burns, 2012, 38, 414-420.

498 7. M. Ahearne, Y. Yang and K. K. Liu, Topics in Tissue Engineering, ed. N. Ashammakhi, R. 499 L. Reis and F. Chielini, Kluwer, Dordrecht, 2008, pp. 1-16.

500 8. M. Ahearne and D. J. Kelly, Biomed. Mater., 2013, 8, 035004.

501 9. W. C. Hayes, L. M. Keer, G. Herrmann and L. F. Mockros, J. Biomech.,1972, 5, 541-551.

502 10. C. Pailler-Matt'ei and H. Zahouani, Tribol. Int., 2006, 39, 12-21.

503 11. K. K. Liu and B. F. Ju, J. Phys. D: Appl. Phys., 2001, 34, 91-94.

504 12. B. F. Ju and K. K. Liu, Mech. Mater., 2002, 34, 485-491.

505 13. M. Ahearne, K. K. Liu, A. J. El Haj, K. Y. Then, S. Rauz and Y. Yang, Tissue Eng., Part 506 C, 2010, 16, 319-327.

507 14. S. L. Wilson, I. Wimpenny, M. Ahearne, S. Rauz, A. J. El Haj and Y. Yang, Adv. Funct. 508 Mater., 2012, 22, 3641-3649. 
510 16. Y. Yang, P. O. Bagnaninchi, M. Ahearne, R. K. Wang and K. K. Liu, J. R. Soc.,

511 Interface, 2007, 4, 1169-1173.

512 17. M. Ahearne, E. Siamantouras, Y. Yang and K. K. Liu, J. R. Soc., Interface, 2009, 6, 471513478.

514 18. K. L. Johnson, Contact Mechanics, Cambridge University Press, Cambridge, Reprint edn, 5151987.

516 19. J. D. Kaufman, G. J. Miller, E. F. Morgan and C. M. Klapperich, J. Mater. Res., 2008, 23, 517 1472-1481.

518 20. M. Eastwood, D. McGrouther and R. Brown, Proc. Inst. Mech. Eng., Part H,1998, 212, $51985-92$.

520 21. J. Fromageau, J.-L. Gennisson, C. Schmitt, R. L. Maurice, R. Mongrainand G. Cloutier,

521 IEEE Trans. Ultrason. Ferroelectrics Freq. Contr., 2007,54, 498-509.

522 22. C. Li, Z. Huang and R. K. Wang, Opt. Express, 2011, 19, 10153-10163.

523 23. A. Samani and D. Plewes, Phys. Med. Biol., 2004, 49, 4395-4405.

524 24. J. Y. Rho, R. B. Ashman and C. H. Turner, J. Biomech., 1993, 26, 111-119.

525 25. B. Hinz, D. Mastrangelo, C. E. Iselin, C. Chaponnier and G. Gabbiani, Am. J. Pathol., $5262001,159,1009-1020$.

527 26. M. A. Griffin, S. Sen, H. L. Sweeney and D. E. Discher, J. Cell Sci., 2004, 117, 58555285863. 
529 27. T. Yeung, P. C. Georges, L. A. Flanagan, B. Marg, M. Ortiz, M. Funaki, N. Zahir, W.

530 Ming, V. Weaver and P. A. Janmey, Cell Motil. Cytoskeleton, 2005, 60, 24-34.

531 28. C. S. Chen, M. Mrksich, S. Huang, G. M. Whitesides and D. E. Ingber, Science, 1997, 532 276, 1425-1428.

533 29. N. L. Halliday and J. J. Tomasek, Exp. Cell Res., 1995, 217, 109-117.

534 30. A. J. Engler, S. Sen, H. L. Sweeney and D. E. Discher, Cell, 2006, 126, 677-689.

535 31. A. Banerjee, M. Arha, S. Choudhary, R. S. Ashton, S. R. Bhatia, D. V. Schaffer and R. S. 536 Kane, Biomaterials, 2009, 30, 4695-4699.

537 32. L. Bian, C. Hou, E. Tous, R. Rai, R. L. Mauck and J. A. Burdick, Biomaterials, 2013, 34, $538 \quad 413-421$.

539 33. A. J. Steward, D. R. Wagner and D. J. Kelly, Eur. Cells Mater., 2013, 25, 167-178.

540 34. B. Trappmann, J. E. Gautrot, J. T. Connelly, D. G. Strange, Y. Li, M. L. Oyen, M. A.

541 Cohen Stuart, H. Boehm, B. Li, V. Vogel, J. P. Spatz, F. M. Watt and W. T. Huck, Nat.

542 Mater., 2012, 11, 642-649.

543 35. A. J. Steward, S. D. Thorpe, T. Vinardell, C. T. Buckley, D. R. Wagner and D. J. Kelly, 544 Acta Biomater., 2012, 8, 2153-2159.

545 36. C. Mauch, B. Adelmann-Grill, A. Hatamochi and T. Krieg, FEBS Lett., 1989, 250, 301546305

547 37. C. T. Buckley, S. D. Thorpe, F. J. O’Brien, A. J. Robinson and D. J. Kelly, J. Mech. 548 Behav. Biomed. Mater., 2009, 2, 512-521. 
549 38. C. T. Buckley, T. Vinardell, S. D. Thorpe, M. G. Haugh, E. Jones, D. McGonagle and D. 550 J. Kelly, J. Biomech., 2010, 43, 920-926.

551 39. J. C. Hu and K. A. Athanasiou, Biomaterials, 2005, 26, 2001-2012.

552 40. T. E. Douglas, P. B. Messersmith, S. Chasan, A. G. Mikos, E. L. de Mulder, G. Dickson, 553 D. Schaubroeck, L. Balcaen, F. Vanhaecke, P. Dubruel, J. A. Jansen and S. C.

554 Leeuwenburgh, Macromol. Biosci., 2012, 12, 1077-1089.

555 41. B. M. Gillette, J. A. Jensen, B. Tang, G. J. Yang, A. Bazargan-Lari, M. Zhong and S. K. 556 Sia, Nat. Mater., 2008, 7, 636-640.

557 42. E. Bell, B. Ivarsson and C. Merrill, Proc. Natl. Acad. Sci. U. S. A., 1979, 76, 1274-1278.

558 43. M. Ahearne, S. L. Wilson, K. K. Liu, S. Rauz, A. J. El Haj and Y. Yang, Exp. Eye Res., $5592010,91,584-591$.

560 44. V. S. Nirmalanandhan, N. Juncosa-Melvin, J. T. Shearn, G. P. Boivin, M. T. Galloway,

561 C. Gooch, G. Bradica and D. L. Butler, Tissue Eng., 2009, 15, 2103-2111.

562 45. Y. Yang, P. O. Bagnaninchi, M. Ahearne, R. K. Wang and K.-K. Liu, J. R. Soc., 563 Interface, 2007, 4, 1169-1173.

564 46. M. E. Fini, Prog. Retinal Eye Res., 1999, 18, 529-551.

565 47. S. L. Wilson, A. J. El Haj and Y. Yang, J. Funct. Biomater., 2012, 3, 642-687.

566 48. J. M. Haus, J. A. Carrithers, S. W. Trappe and T. A. Trappe, J. Appl. Phys.,2007, 103, $567 \quad 2068-2076$.

568 49. S. L. Wilson, M. Guilbert, J. Sul'e-Suso, J. Torbet, P. Jeannesson, G. D. Sockalingum and 569 Y. Yang, FASEB J., 2013, DOI: 10.1096/13-227579. 
570 50. M. Ahearne, Y. Yang, A. J. El Haj, K. Y. Then and K. K. Liu, J. R. Soc.,Interface, 2005, 5712 2, 455-463.

572 51. R. A. Brown, M. Wiseman, C. B. Chuo, U. Cheema and S. N. Nazhat, Adv.Funct. Mater., $5732005,15,1762-1770$.

574 52. L. A. Micol, M. Ananta, E.-M. Engelhardt, V. C. Mudera, R. A. Brown,J. A. Hubbell and 575 P. Frey, Biomaterials, 2011, 32, 1543-1548.

576 53. J. V. Cauich-Rodriguez, S. Deb and R. Smith, Biomaterials, 1996, 17,2259-2264.

577 54. R. Sunyer, A. J. Jin, R. Nossal and D. L. Sackett, PLoS One, 2012, 7, e46107.

578 55. O. Ariga, M. Kato, T. Sano, Y. Nakazawa and Y. Sano, J. Ferment. Bioeng.1993, 76, 579 203-206.

580 56. N. E. Vrana, A. Elsheikh, N. Builles, O. Damour and V. Hasirci, Biomaterials, 2007, 28, $581 \quad 4303-4310$.

582 57. J. Torbet, M. Malbouyres, N. Builles, V. Justin, M. Roulet, O. Damour,A. Oldberg, F. 583 Ruggieo and D. J. S. Hulmes, Biomaterials, 2007, 28, 4268-4276.

584 58. O. Valiron, L. Peris, G. Rikken, A. Schweitzer, Y. Saoudi, C. Remy and D. Job, J. Magn. 585 Reson. Imag., 2005, 22, 334-340.

586 59. W. E. Teo and S. Ramakrishna, Nanotechnology, 2006, 17, 89-106.

587 60. S. L. Wilson, I. Wimpenny, M. Ahearne, S. Rauz, A. J. El Haj and Y. Yang, Adv. Funct. 588 Mater., 2012, 22, 3641-3649.

589 61. K. Tonsomboon and M. L. Oyen, J. Mech. Behav. Biomed. Mater., 2013, 21,185-194. 


\section{Figures captions and captions}

592 Figure 1. Schematic representation of the following mechanical tests where $F$ represents the

(A)

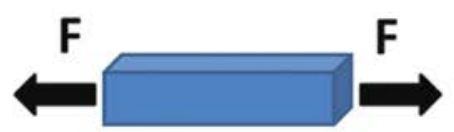

(E)

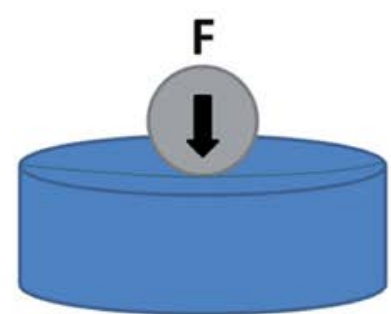

(B)

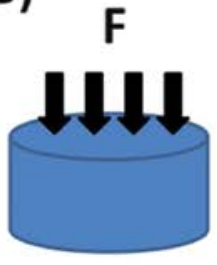

(C)

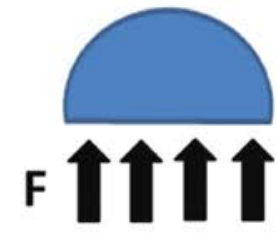

(D)

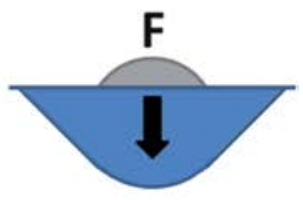

(F)

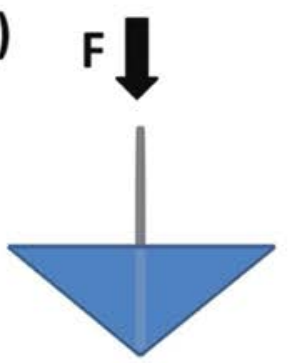

(G)

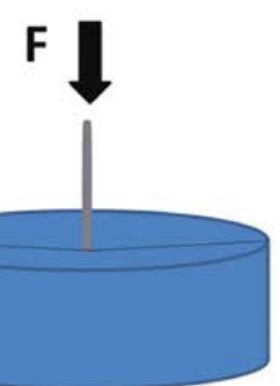

593 applied force: (A) tensile; (B) compression; (C) inflation; (D) spherical indentation

594 (suspended); (E) spherical indentation (on substrate); (F) micro-indentation (suspended); (G)

595 micro-indentation (on substrate).

596 


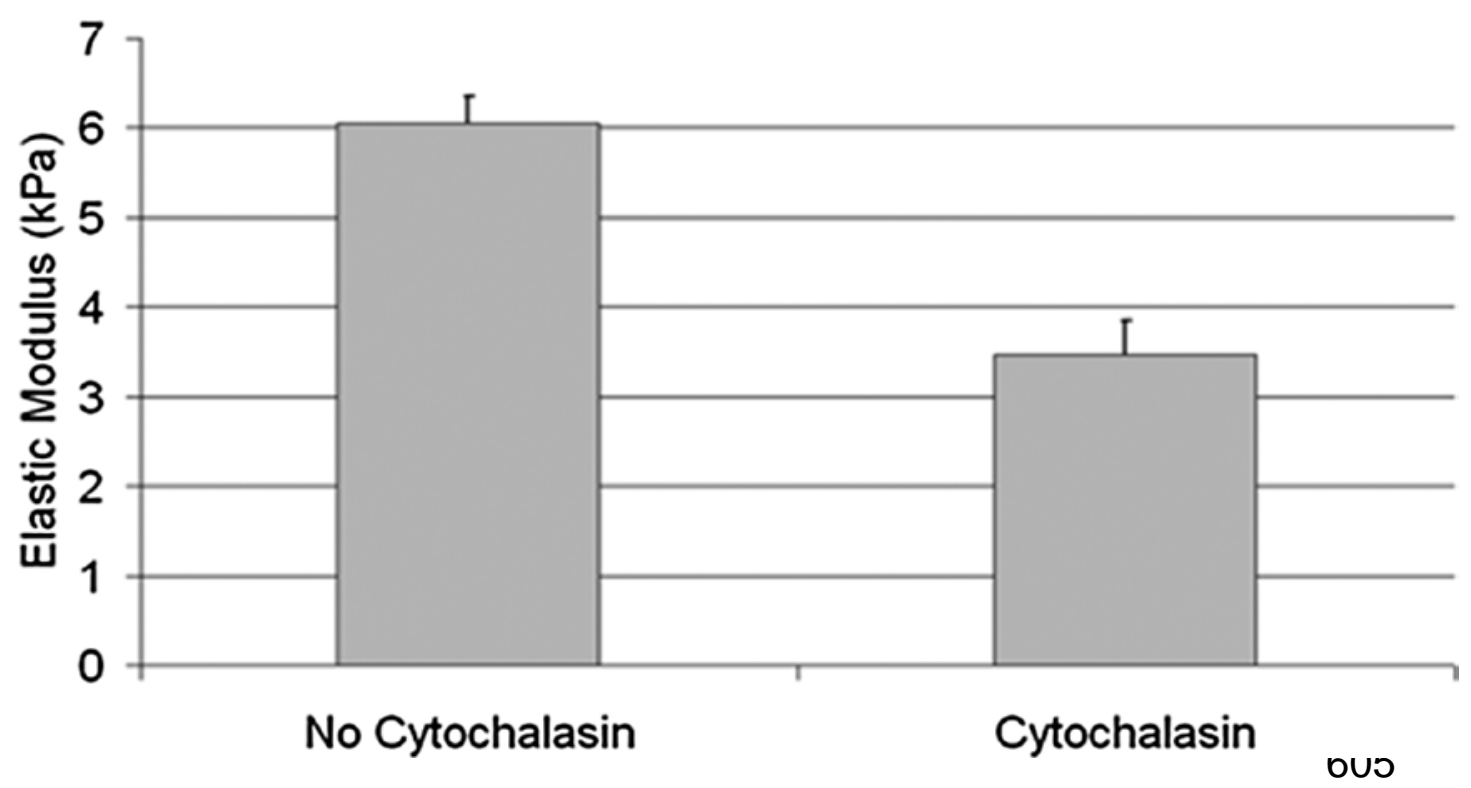

606 Figure 2 .The elastic modulus change in response to the addition of cytochalasin in collagen 607 hydrogel seeded with fibroblasts, which disrupted actin filament in fibroblasts.(13) 608 


\section{Day 0}

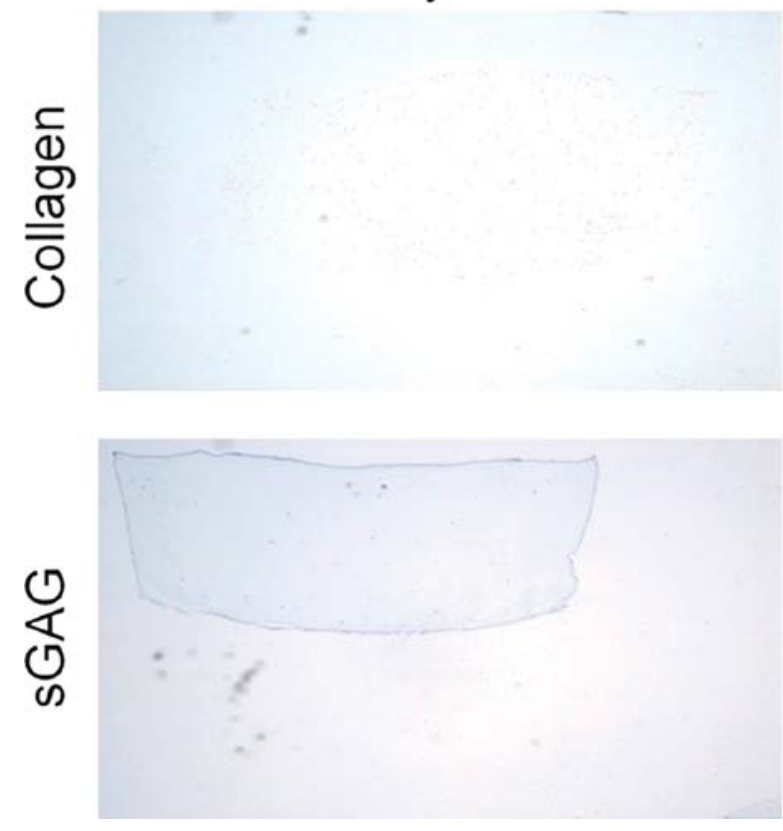

Day 21
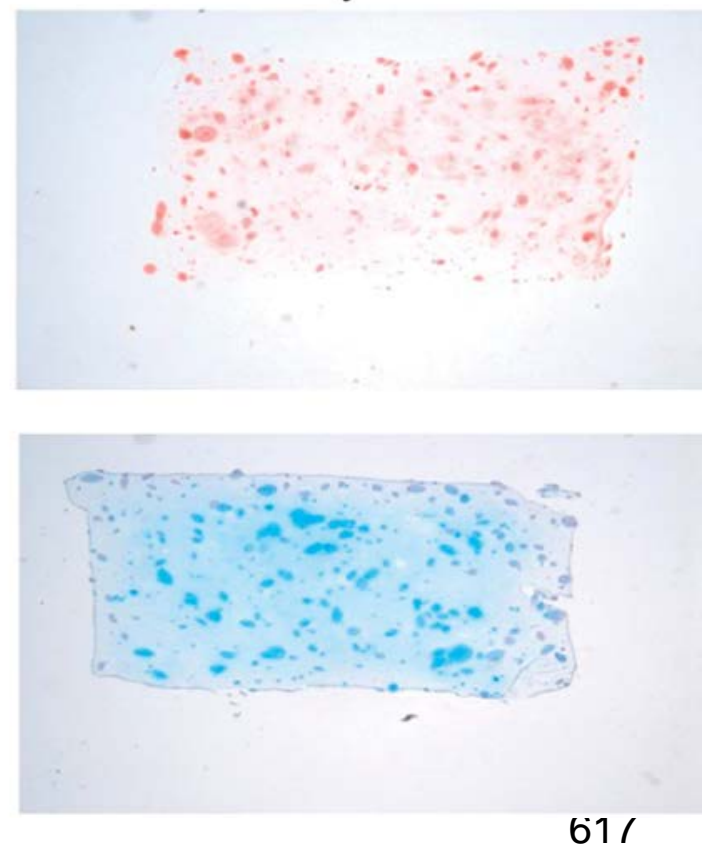

618 Figure 3. Increase in collagen (stained with picrosirius red) and sGAG (stained with Alcian

619 blue) in cell-seeded agarose hydrogels after 21 days in culture in a chondrogenic medium 620 


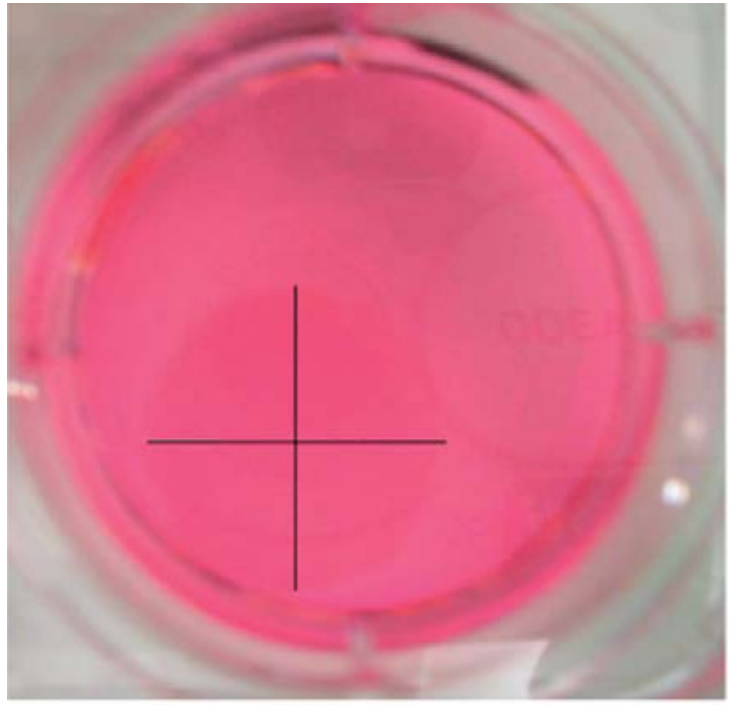

1 hour

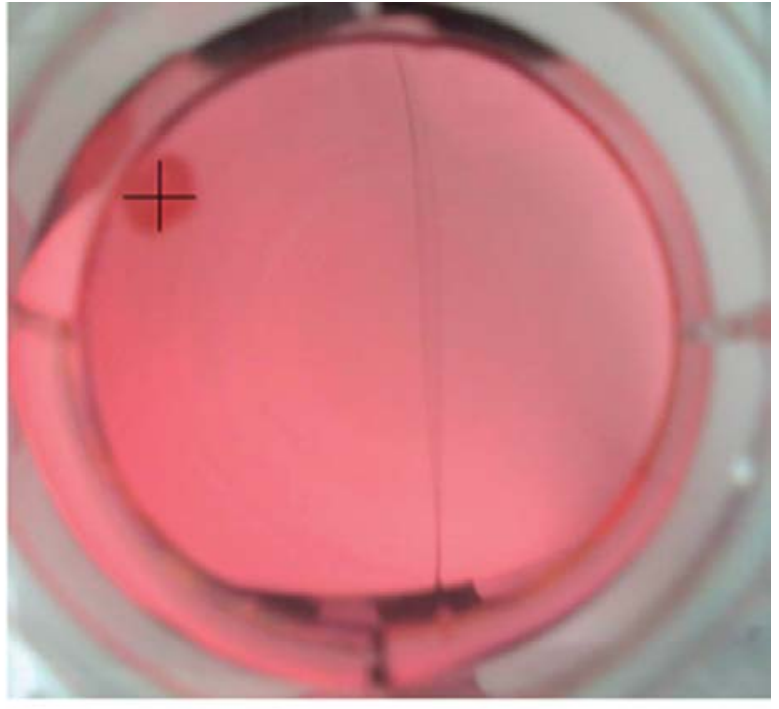

\section{5 days}

622 Figure 4: Images of a collagen hydrogel seeded with corneal fibroblasts that have undergone 623 contraction.(13)

624 

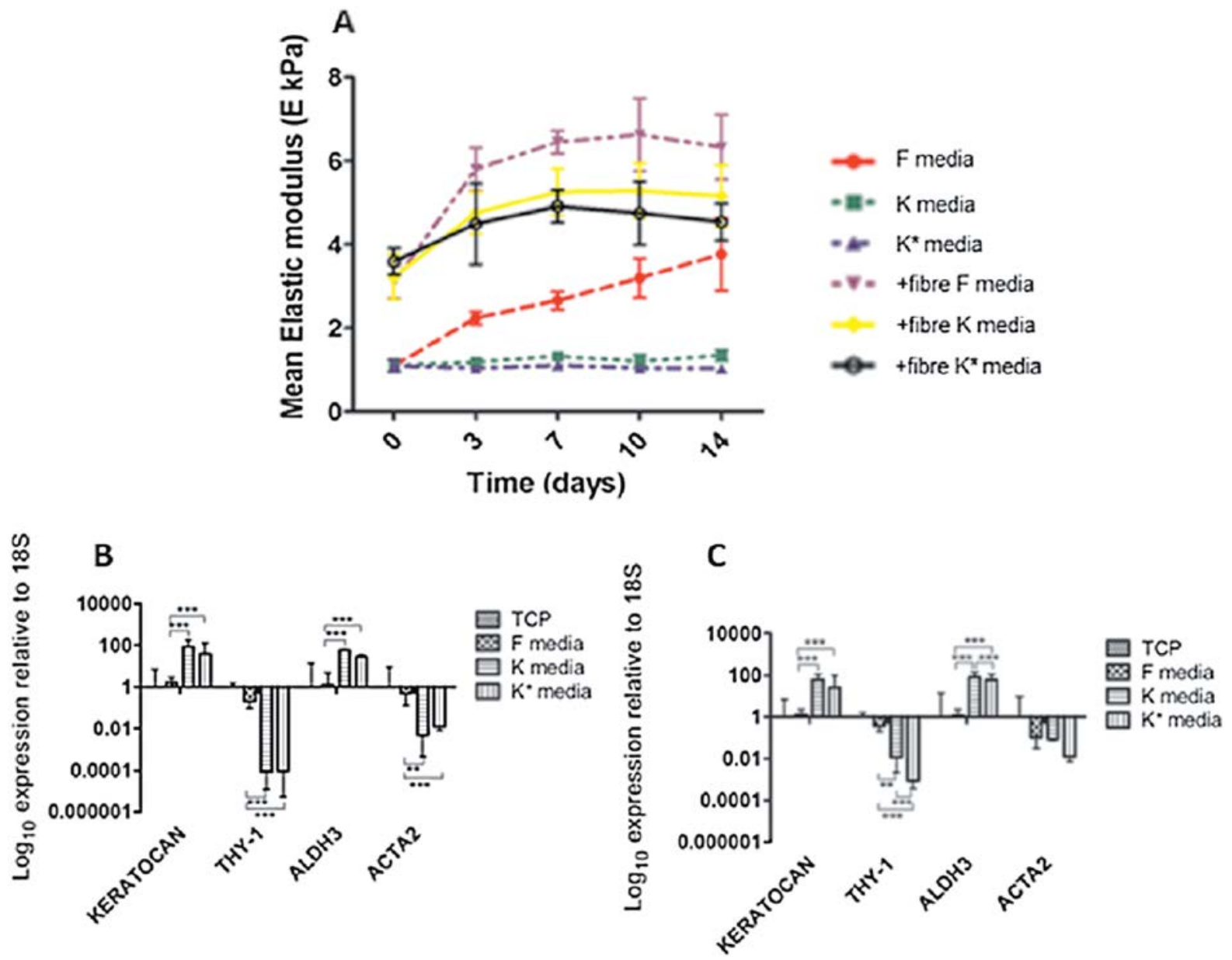

626 Figure 5. (A) The elastic modulus and gene expression of corneal stromal cells grown in

627 collagen hydrogels for 14 days in response to chemical and topographic regulation. F denotes 628 serum-containing medium, K denotes serum-free, insulin supplemented medium, and K0

629 denotes serum-free b-FGF supplemented medium; +fibre indicates the incorporation of

630 nanofibres in the hydrogel. (B) The gene expression without nanofibre incorporation; (C) the

631 gene expression with nanofibre incorporation; keratocan and ALDH3 are keratocyte-specific

632 genes, while Thy-1 and AQ2 ACTA2 are corneal fibroblast-specific genes 


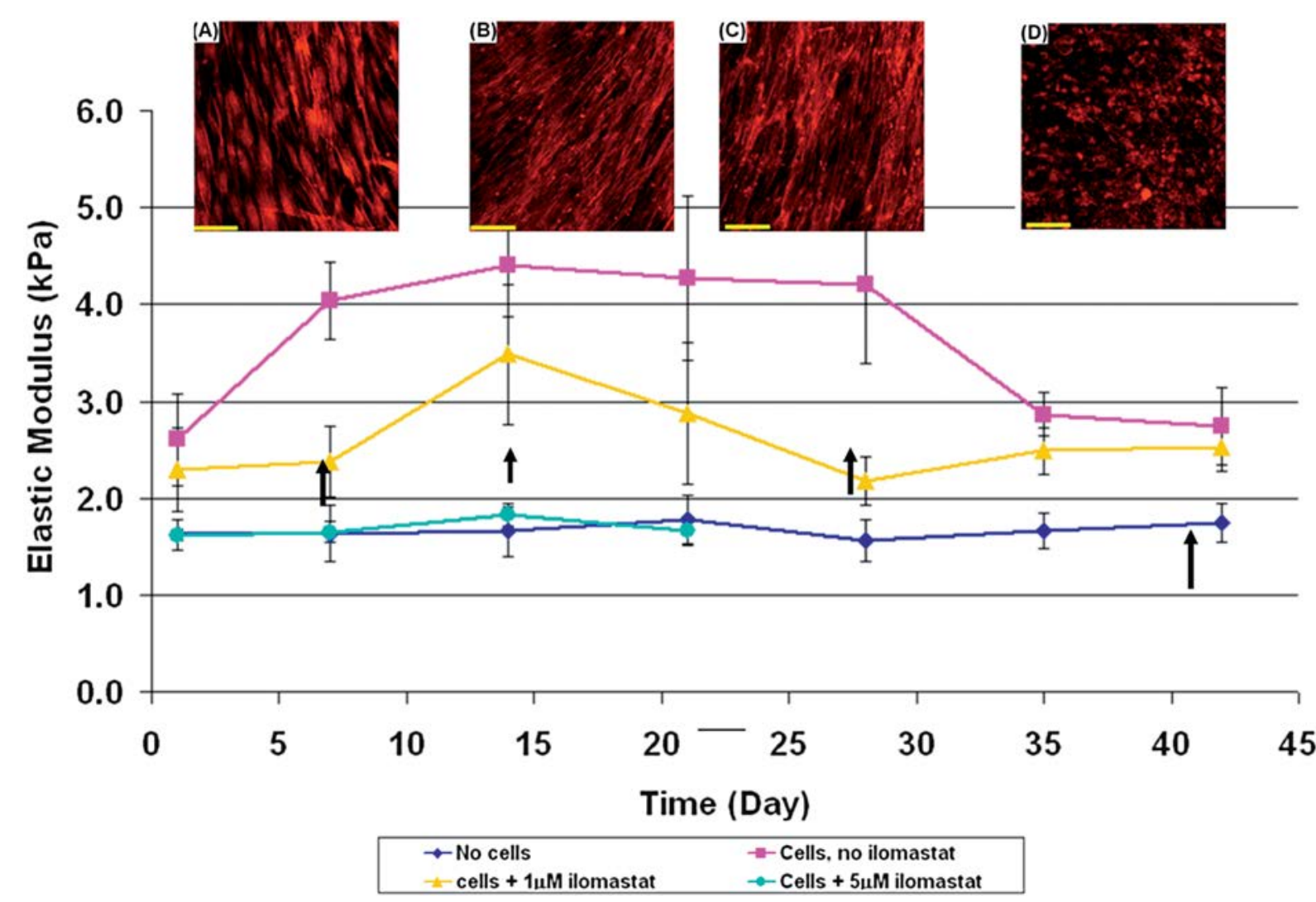

634

635

636 Figure 6: Change in elastic modulus of collagen hydrogels seeded with corneal stromal

637 fibroblasts in response to culture time and the MMP inhibitor ilomastat.(13) Corresponding 638 actin stained specimens at (A) 7 days; (B) 14 days; (C) 21 days and (D) 42 days 


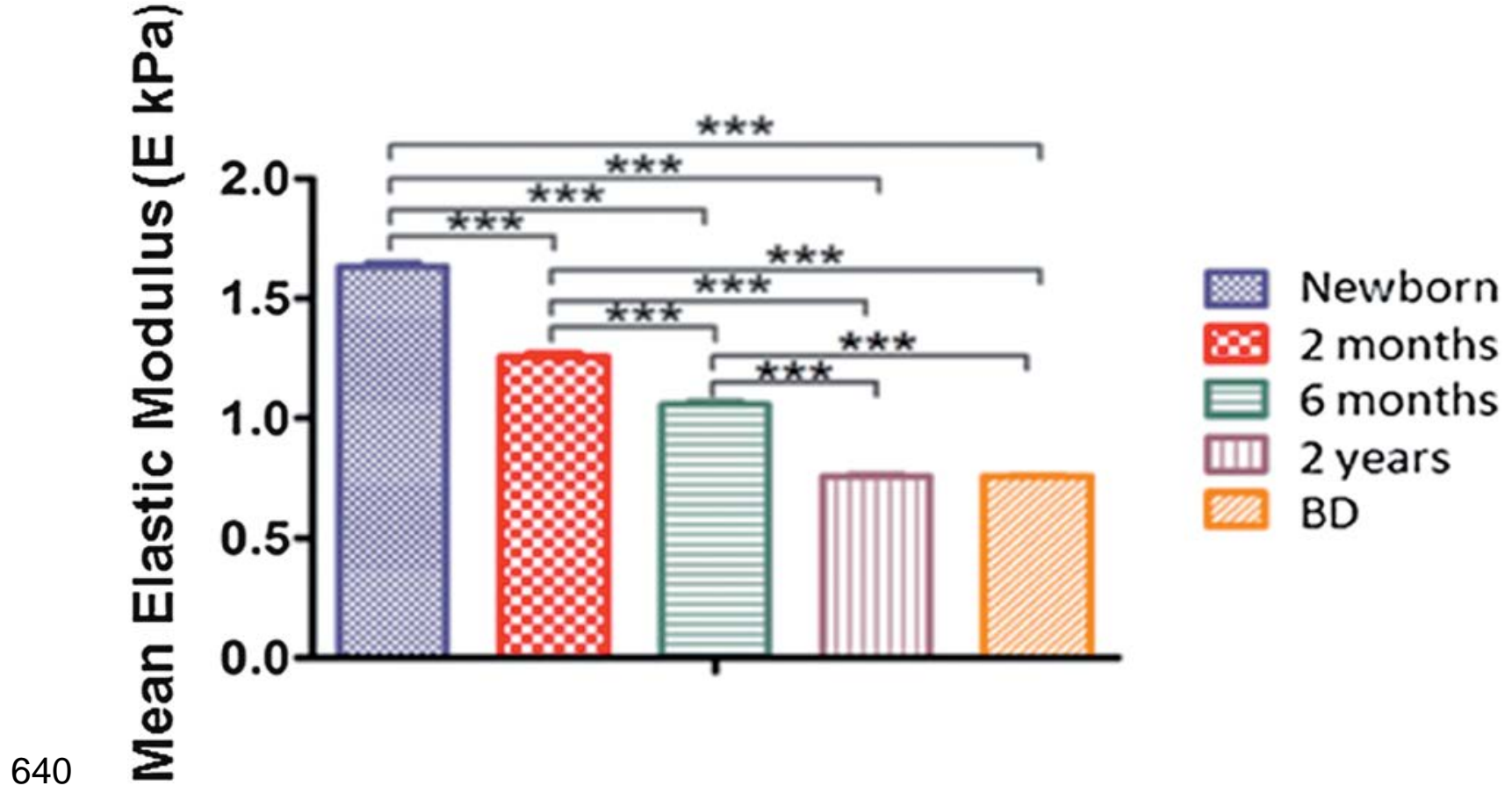

641

642 Figure 7. The elastic modulus of acellular hydrogel scaffolds using collagen extracted from 643 rats of different ages.

644 


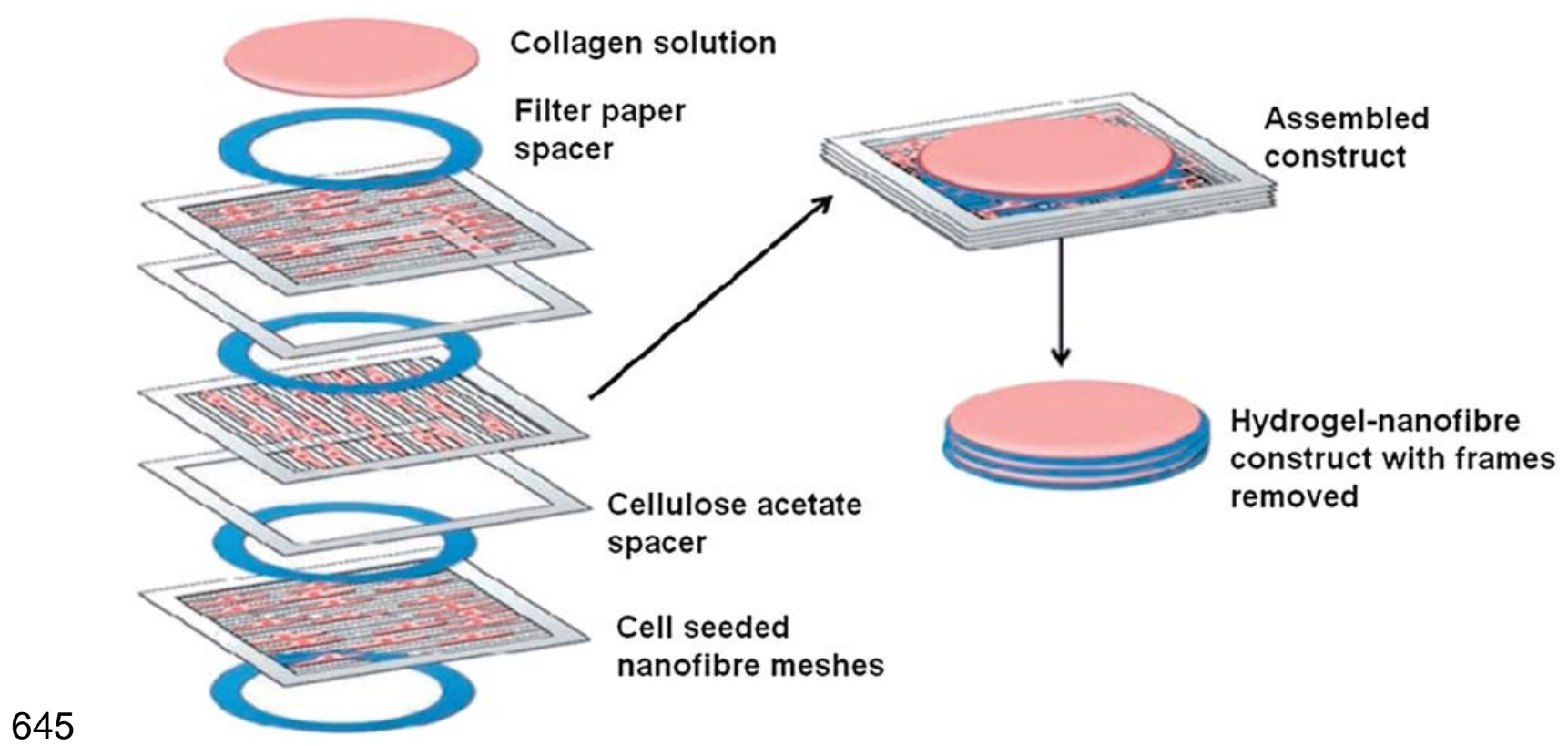

646 Figure 8. Schematic representation of the assembly process used to fabricate a nanofibre 647 hydrogel construct 